\title{
INVESTIGATION OF PERFORMANCE AND LANE UTILIZATION WITHIN A PASSING LANE ON A TWO LANE RURAL HIGHWAY
}

\author{
Zachary Freedman ${ }^{1}$, Ahmed Al-Kaisy ${ }^{2}$ \\ ${ }^{1}$ Sabra, Wang \& Associates, Baltimore, USA \\ ${ }^{2}$ Civil Engineering Department, Montana State University, USA
}

Received 18 October 2012; accepted 6 June 2013

\begin{abstract}
An investigation into platooning and passing maneuvers within a passing lane section on a rural two-lane, two-way highway was considered in this study. The study site was located on US Highway 287 between the town of Townsend and the City of Helena in the state of Montana. Traffic volumes at study site, while considered relatively low, were typical on two-lane highways in many rural states. Per-lane analysis of performance measures and lane utilization (volume split) were used to indirectly examine passing maneuvers and lane changing at successive locations within the passing lane section. For the case study site, it was evident that traffic performance became relatively stable beyond half a mile into the passing lane for the traffic volumes investigated. Therefore, results strongly suggested that most passing maneuvers already took place before the 0.5 -mile station and that the actual passing lane length was well beyond the optimal length required for breaking up platoons and improving performance.
\end{abstract}

Keywords: platooning, passing lanes, two-lane highways, performance.

\section{Introduction}

Two-lane highways constitute the vast majority of highway facilities particularly in states where most vehicular travel takes place in rural areas. Passing maneuvers are restricted on those highways and are typically performed using the opposing lane when sight distance and gaps in the opposing traffic stream permit. This has serious implications on traffic performance and safety. From traffic performance perspective, the limited passing opportunities would result in higher impact of slow-moving vehicles on mobility and performance. This impact generally increases with the increase in traffic level in the two directions of travel, the proportion of slow-moving vehicles in the traffic stream, and the average speed differential between the mainstream and slower vehicles. The use of passing lanes is known to alleviate this unique operational characteristic on welltraveled two-lane highways. Passing lanes allow vehicles traveling at faster speeds to overtake slow-moving vehicles, thus breaking up platoons and reducing delays associated with inadequate passing opportunities. Therefore, it is well known that using passing lanes generally improves operations and results in better level of service on twolane highways. Two very important aspects in planning and designing passing lanes on two-lane highways are the determination of the appropriate passing lane length and the frequency at which passing lanes are provided. This study examines platooning and

${ }^{1}$ Corresponding author: zfreedman@sabra-wang.com 
performance within the passing lane, which is important in understanding the length requirement for passing lanes on two-lane highways.

\section{Literature Review}

In the course of the literature review performed in this study, only limited research was identified that is related to passing lane length requirement. In this section, information available about passing lane length requirement in the current practice as well as the few relevant studies in the literature are presented.

The Highway Capacity Manual (HCM) (TRB, 2010) recommends a passing lane length based on traffic level as shown in Table 1. The source of the values provided in this table is a study that was conducted more than 25 years ago using traffic simulation (Harwood, 1987).

Table 1

Optimal Passing Lane Length in the HCM

\begin{tabular}{|l|l|}
\hline $\begin{array}{l}\text { Directional Demand } \\
\text { Flow Rate (pc/h) }\end{array}$ & $\begin{array}{l}\text { Optimal Passing Lane } \\
\text { length }(\mathbf{m i})\end{array}$ \\
\hline$\leq 100$ & $\leq 0.5$ \\
$>100 \leq 400$ & $>0.5 \leq 0.75$ \\
$>400 \leq 700$ & $>0.75 \leq 1.0$ \\
$\geq 700$ & $>1.0 \leq 2.0$ \\
\hline
\end{tabular}

Source: TRB (2010)

The Policy on Geometric Design of Highways and Streets (AASHTO, 2004), known as the Green Book, suggests values for passing lane length that are very consistent with the values in the current HCM. The absolute minimum length for passing lanes according to the Green Book is $300 \mathrm{~m}$ (1000 ft.) excluding tapers. Also, the optimal length provided in the green book is $0.8-3.2 \mathrm{~km}$ (0.5-2.0 miles) which is the same range of values provided in Table 1 .

A few researchers have investigated performance and/or passing maneuvers within passing lanes with the objective of understanding the passing lane length requirement under various traffic conditions.

Harwood and Hoban (1987) used the traffic simulation model TWOPAS in an attempt to determine optimal length and spacing of passing lanes. Their data showed that optimal passing lane length was dependent on traffic volume. For low traffic level, the optimal passing lane length is 0.5-0.75 mile. For medium traffic level (around 400 vehicles per hour) the optimal length is $0.75-1.0$ mile, and for higher traffic level (equal or greater than 700 vehicles per hour) the optimal length is 1.02.0 miles. Results from this study were used in developing the HCM recommended values for passing lane lengths in the HCM 2000 and HCM 2010 (TRB, 2000; TRB, 2010).

Another study by Harwood and St. John (1984) investigated twelve passing lane sites in six states. The purpose of this study was to determine the effective length and spacing of passing lanes. At each site, automatic traffic recorders and observers were used to collect data. The data recorders collected vehicle mix, speed, headway, and acceleration while the observers primarily counted the passing maneuvers. The locations of the data recorders were: 500 feet upstream of the entrance taper, 100 feet downstream of the entrance taper, in the middle of the passing lane, 300 feet from the beginning of the exit taper, 500 feet downstream of the exit taper, and $1 \mathrm{mi}$ downstream of the exit taper. Six hours of data were collected at each site. The main measures of effectiveness for this study were traffic speed, percent of vehicles in a platoon and passing rate. The study found that operational benefits of passing lanes exist over a distance of three to eight miles and that the optimal length of passing lanes generally vary from 0.5 mile to 1.0 mile. 
Woolridge et al. (2002) researched the optimal length and spacing of passing lanes on two lane highways using the traffic simulation model TWOPAS. A generic two-lane highway with varying length of passing lane was tested. The spacing between passing lane sections was varied between 1 and $8 \mathrm{mi}$ at $1 \mathrm{mi}$ intervals and the length of passing lane was varied between 0.25 and $2 \mathrm{mi}$ at $0.25 \mathrm{mi}$ intervals. The results of the simulation showed that the optimal length of passing lane varied between 0.8 mile and 2.0 miles and the optimal spacing varied between 3.5 miles and 11 miles.

Gattis et al. (2006) studied sites with a 2+1 passing lane configuration (also known as a three-lane configuration or an alternating passing-lane) in Arkansas. This configuration has one lane in each direction (like a normal two-lane highway) and center lane that acts as a passing lane alternating in direction. This is demonstrated in Fig. 1.

Four sites were selected for study of platoonbreakup and passing rates: two on US-70, one on US-65, and one on US-82. Study results showed that the greatest reduction in platooning was between 0.93 miles and 1.86 miles into the three-lane section and that the platooning at the beginning of the three-lane segment was always greater than at the end. Further, the researchers found that the maximum passing rate occurred between 0.93 miles and 1.86 miles into the site. Past 1.86 miles, passing rate leveled off or declined. Passing rates also increased with higher volumes.

Emoto and May (1988) conducted a field study to analyze the passing lane usage. A plot of the total volume versus the passing lane volume was created. A best fit line was developed and a passing experiment was conducted to determine the number of passes vehicles made per mile of passing lane. The number of passes varied from 1.5 passes per mile to 1.75 passes per mile.

\section{Problem Statement}

The periodic use of passing lanes is one of the most common operational improvements on two-lane highways. In many instances, it is proven more feasible than other costlier improvements such as the super 2-plus- 1 and the upgrade to a four-lane highway facility. In planning and designing passing lanes, two parameters are deemed very important; the passing lane length required and the interval at which the passing lane should be provided on a particular highway to achieve the desired performance improvement. This current research is associated with the former of the two design parameters.

The determination of the needed or optimal passing lane length requires a good

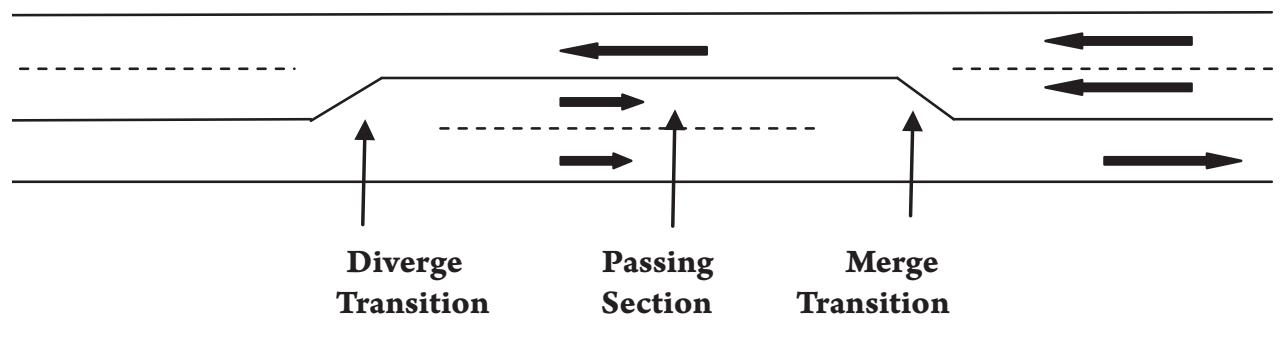

Fig. 1.

2+1 Passing Lane Configuration 
understanding of traffic behavior within the passing lane sections. Developing such an understanding is somewhat challenging in that it should largely rely on empirical field observations, particularly in the absence of advanced and proven simulation models. Vehicular interactions in most simulation models are based on highly theoretical and sometimes very simplistic car-following and lane changing models that may not yield realistic results. This is especially true for the simulation of two-lane highway operations where only a limited number of simulation models are available to researchers and practitioners. On the other hand, field investigations are constantly challenged, to various degrees, by limitations to site geometrics, traffic conditions, data collection techniques, or combinations of the aforementioned factors. Nonetheless, field observations offer valuable information about the dynamics of vehicular interactions within the passing lane section that is much needed in the literature. As such, it is the objective of this research is to empirically explore the dynamics of platoon break-up within a typical passing-lane section with respect to the passing lane length requirement. While this case study involved a single passing lane site, findings of the case study is deemed applicable to many similar sites on two-lane highways in rural areas.

\section{Materials and Methodology}

\subsection{Study Site}

The study site investigated in this research is located on US Highway 287 Southbound between the town of Townsend and the City of Helena in the state of Montana. This particular passing lane is roughly located at around 8 miles north of Townsend and 25 miles south of Helena. The passing lane is around 2.33 miles in length and is located in level terrain. The highway has standard lane width and wide shoulders at the passing lane location. No major access points exist which could affect travel patterns within the passing lane of interest. Only one unpaved driveway in the Northbound direction exists and is located further downstream in the study area. Southbound traffic using the driveway was observed in the field and found too small to have any noticeable influence on lane utilization patterns. The Annual Average Daily Traffic (AADT) at this site in the data collection year (2008) was around 5000 vehicles per day.

\subsection{Study Design}

In order to investigate performance and lane utilization, multiple detector stations were used at the study site. Specifically, five automatic traffic recorder stations were used to gather data both upstream and within the passing-lane over a one week period in June 2008. As illustrated in Fig. 2, the first station was located 250 feet upstream of the passinglane; the other four stations were located at 0.25 mile, 0.5 mile, 1.0 mile, and 1.5 miles downstream of the lane addition taper into the passing-lane. Traffic data including counts, speeds, headways, and vehicle classification were obtained at the single lane upstream of the passing lane and for each individual lane within the passing lane section. Counts and vehicle classification were used to establish lane utilization within the passing lane while headways, speeds, classification and counts were used in investigating performance upstream and within the passing lane section. Three different performance measures were used in the study; percent followers, average travel speed, and follower density. Percent followers represents the percentage of vehicles with short headways (less than 3 seconds) 
in the traffic stream. This is the surrogate measure used by the HCM for estimating percent-time-spent following in the field. Follower density is the number of followers in a directional traffic stream over a unit length, typically one kilometer or one mile. It can easily be found by multiplying percent followers and traffic density. This measure, which has been used in South Africa (Van As, 2003), was found the most promising among other measures investigated in a recent study (Al-Kaisy and Karjala, 2008). Average travel speed is the arithmetic mean of all speeds observed at a point location and is the secondary performance measure used by the HCM for class I two-lane highways, which includes rural two-lane arterials and primary highways (TRB, 2010).

\section{Study Results}

\subsection{Per Lane Examination of Performance Measures}

In order to understand traffic behavior within the passing lane, performance measures most relevant to platooning and impedance were examined individually on the normal and passing lanes. As discussed earlier, three measures of performance were examined in this analysis; percent followers, follower density, and average travel speed. In this analysis, plots of each performance measure were created as a function of distance into the passing lane at different traffic levels.

Fig. 3 shows the percent followers at successive data collection stations within the passing lane section at three different traffic levels. On the normal (left) lane, the percent followers seemed to rise significantly in the first 0.25 mile, then dropped to or slightly below its original level (upstream of passing lane) between 0.25 mile and 0.5 mile on that same lane and finally leveled off. On the passing (right) lane, the changes exhibited a different pattern: percent followers remained relatively steady in the first 0.25 mile, slightly dropped between 0.25 and 0.5 mile, and eventually leveled off. These patterns are common to all traffic levels investigated. One observation that was somewhat unexpected here was the relatively low reduction in percent followers in the passing lane section despite the fact that the total traffic on the single lane has split over two lanes within the passing section. In fact, percent followers at 0.25 mile station was much higher than the original value when averaged over the two lanes. It should be understood that, those results did not suggest deterioration in performance upon entering the passing lane, but rather a sign of the limitation in using the time headway as the only performance indicator.

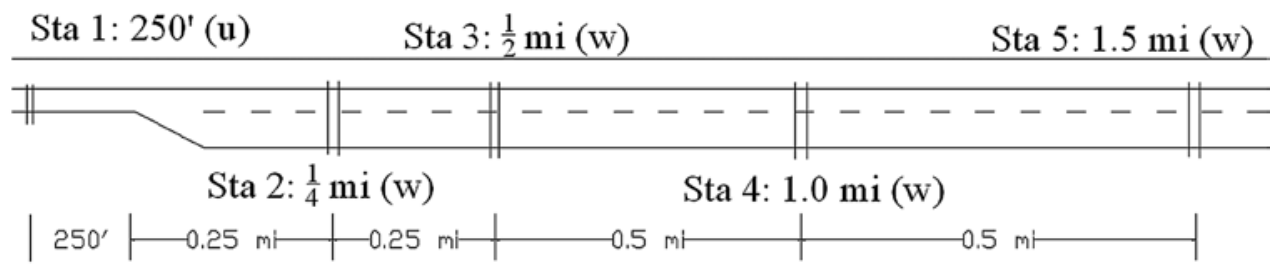

Fig. 2.

Field Data Collection Set-Up 


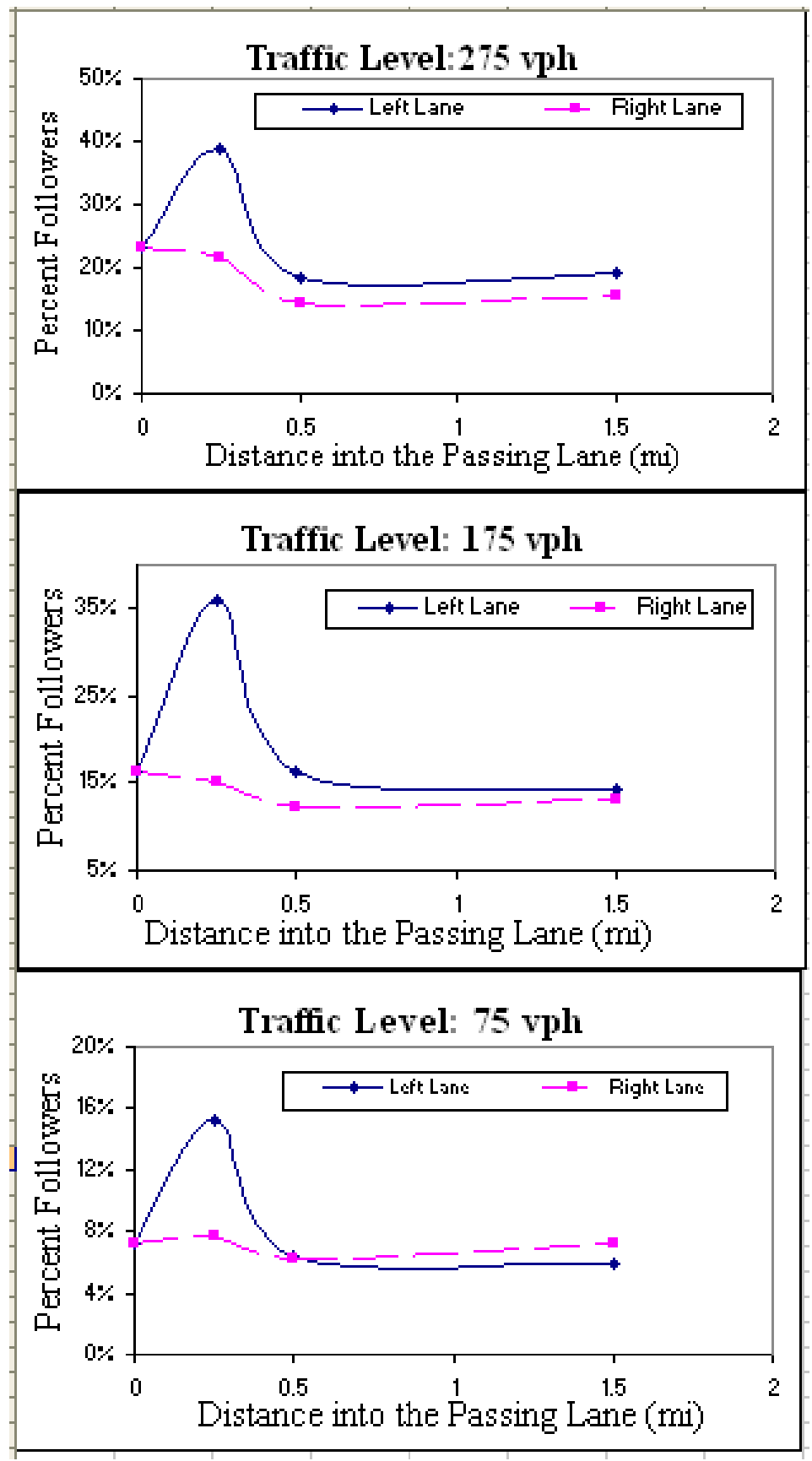

Fig. 3.

Percent Followers at the Passing Lane Section for Various Traffic Levels 
Fig. 4 shows the follower density at successive data collection stations within the passing lane section at the same different traffic levels. As shown, follower density in each lane dropped from the pre-passing-lane value within the first 0.25 mile, in spite of the rise in percent followers. This is because traffic volume split into two separate volumes in the two available lanes beyond the taper. Apparently, the effect of the decrease in volume on follower density was higher than that of the increase in percent followers at 0.25 mile station, which was clearly exhibited in Fig. 3. At 0.25 mile into the passing lane, follower density in the left lane was significantly higher than that in the right lane. This may well be related to the notable increase in percent followers at this station as illustrated in Fig. 3. The only exception was when traffic level was at around $75 \mathrm{vph}$. At this traffic level, it seemed that the density in the normal lane has decreased to an extent that caused follower density on the left lane to be lower than that on the right lane regardless of the increase in percent followers. At 0.5 mile and 1.5 mile into the passing lane section, follower density was always higher on the passing lane than on the normal lane. In general, the lower the traffic level, the higher the discrepancy in follower density between the two lanes. Given the relatively close values for percent followers at the 0.5 -mile and 1.5 -mile stations, results shown in this figure indicated that more traffic use the right lane in general, including free moving vehicles outside vehicular platoons which constitute high proportion of vehicles at low traffic levels.

Fig. 5 shows average travel speed on the two lanes within the passing section at various traffic levels. Speed changes along the distance into the passing lane exhibited very consistent trends at all traffic levels particularly in the normal (left) lane. In this lane speed increased sharply in the first 0.5 mile (around 13\% increase), then declined to a lower value at 1.5 mile into the passing lane. The final speed at 1.5 mile distance was still notably higher than the original prepassing lane speed. Specifically, the speed at 1.5-mile distance was roughly the middle value between the pre-passing lane speed and that at 0.5 mile distance into the passing lane. A totally different trend is shown for the other lane. In the passing lane, speed generally maintained its pre-passing lane value with the exception of a small increase (around $2 \mathrm{mph}$ ) between 0.25 mile and 0.5 mile into the passing lane. As expected, the final speed in the passing lane at 1.5 mile distance was lower than its counterpart for the normal lane, but was still higher than the pre-passing lane speed.

The previous results reveal some of the important aspects of drivers' behavior in using passing lanes. Upon arriving at the passing lane section, traffic was normally split into two streams, one in each travel lane. Examining performance measures in Figs. 3-5 suggest that aggressive drivers always opted to remain in the normal (left) lane, while slower moving vehicles and other vehicles that are not much affected by slower speeds tended to use the passing lane. The sharp increase in short headways in the normal lane (as evidenced by the percent followers at 0.25 mile in Fig. 3 ) and the notable increase in speed (Fig. 5) both suggest that the majority of aggressive drivers used the left lane upon arriving at the passing lane section. On the other hand, changes on the passing lane are less notable. Percent follower slightly declined and average speed slightly increased (around 4\%) mainly because of the drop in traffic flow from the pre-passing lane level.

\subsection{Per Lane Analysis of Traffic Volumes}

To gain further insights into traffic behavior within the passing lane section, and to better 


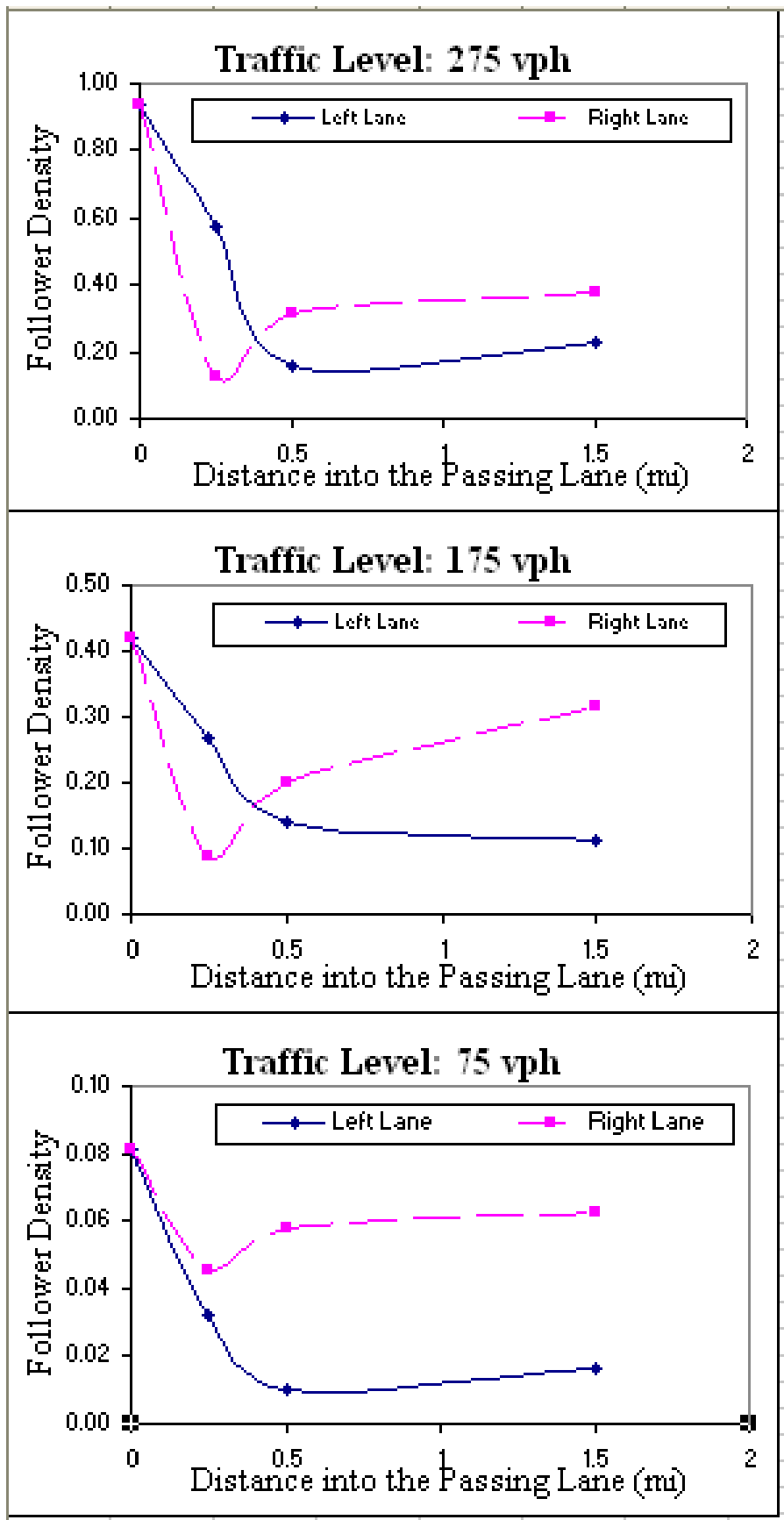

Fig. 4.

Follower Density by Lane at the Passing Lane Section 


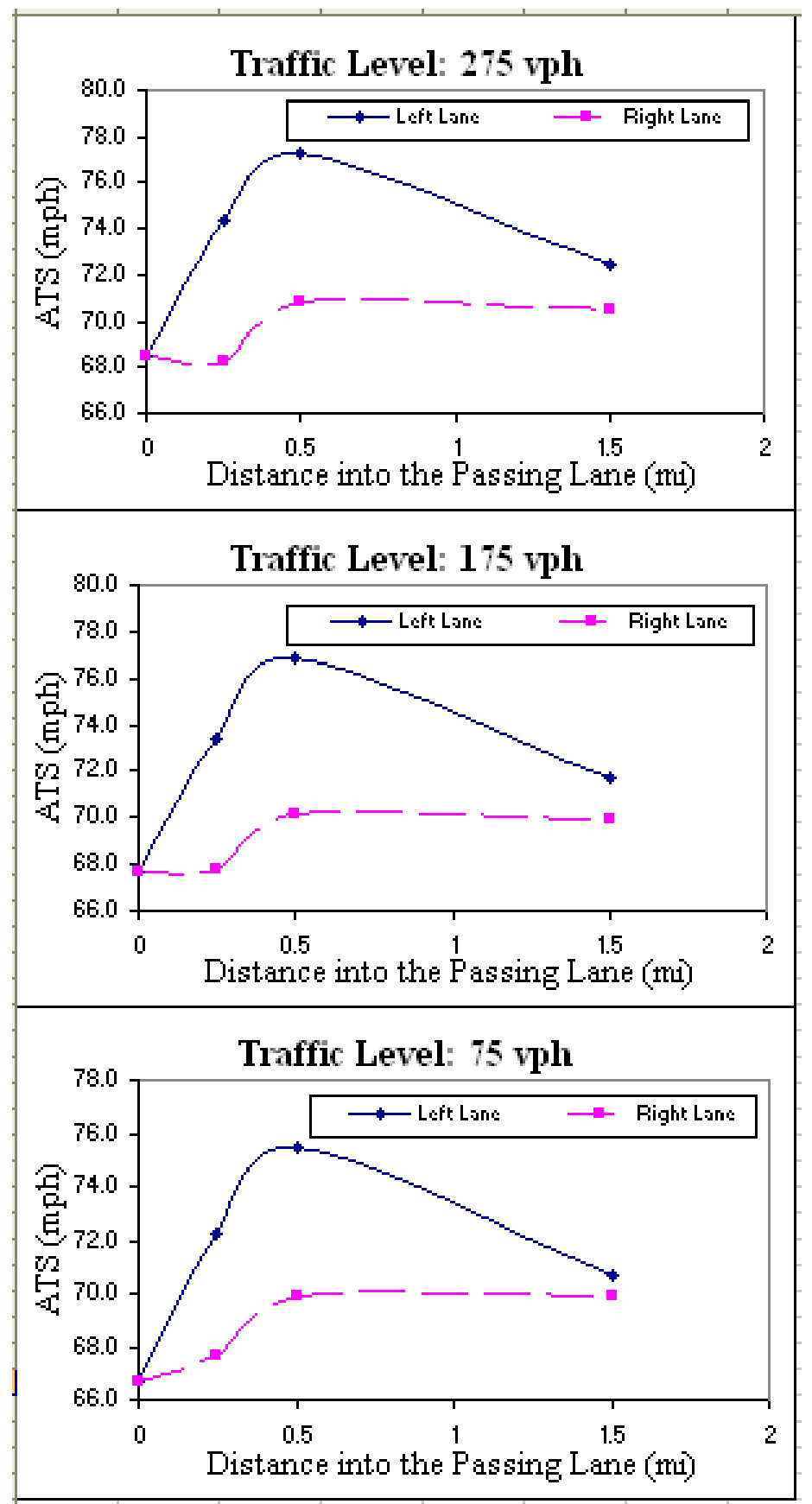

Fig. 5.

Average-Travel-Speed at the Passing Lane Section 
understand the performance trends discussed in the previous section, traffic counts at the successive data collection stations are analyzed at various traffic levels and results are presented in Fig. 6. This figure shows traffic volume split in the two available lanes as a percentage of the total volume at the data collection stations. As shown in this figure, the majority of traffic chose to use the passing lane within the distance investigated. The analysis shows that, at 0.25 -mile distance, between $60 \%$ and $80 \%$ of traffic utilized the right (passing) lane depending on the traffic level. At 0.5 -mile distance into the passing lane, these percentages increase and they all fall roughly in the range of $70 \%$ to $85 \%$. This indicates that many drivers may have chosen to change lanes beyond the taper section and well into the passing lane section. One possible explanation could be as follows: some moderate (non-aggressive) drivers stuck behind slow moving vehicles may have chosen to take the right lane, but only after passing the vehicle(s) they were impeded by, while others may have made the same decision when caught up by aggressive drivers traveling at higher speeds in the left lane. Besides the increase in passing lane split, this hypothesis

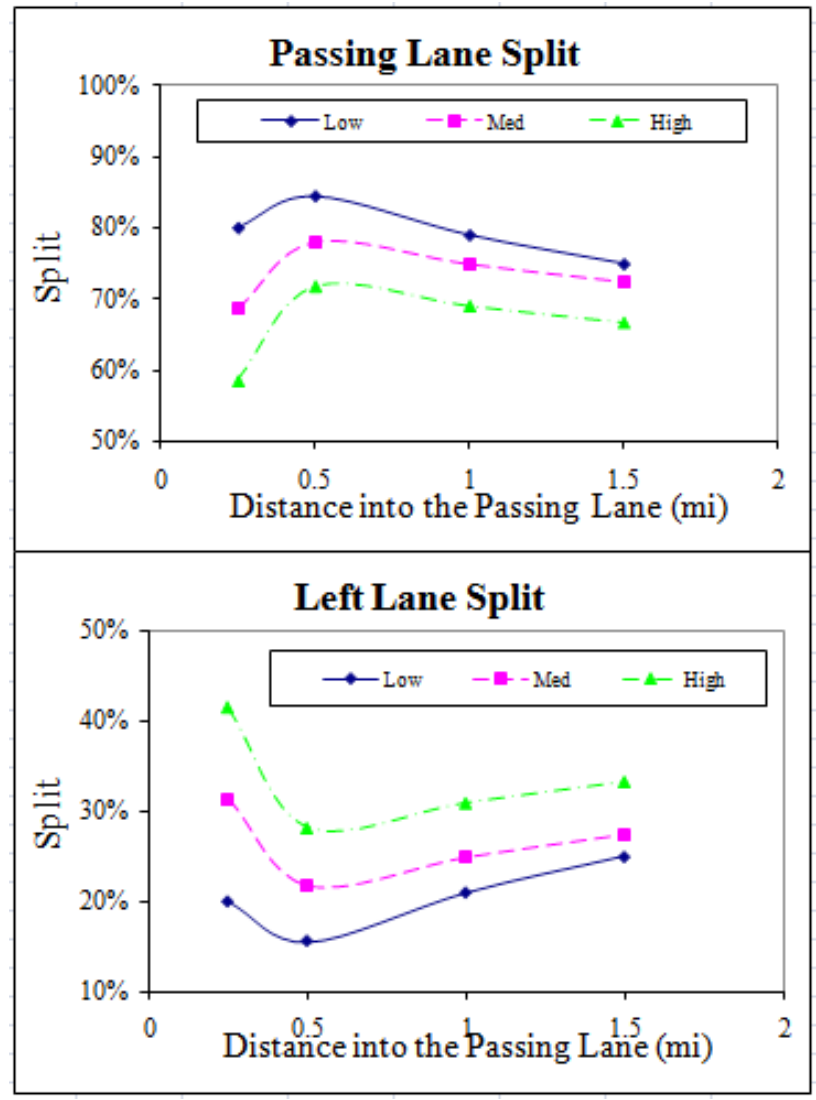

Fig. 6.

Volume Split over Available Lanes at the Passing Lane Section for Various Traffic Levels 
is also supported by the increase in average travel speed and the slight decrease in percent followers in the passing lane due to these moderate drivers moving into the passing lane.

At 1.5-mile distance, the passing lane split slightly declined and varied in the range between $65 \%$ and $75 \%$ depending on the traffic level. This indicates that some drivers may have decided to return to the normal lane well in advance of the passing lane end. This is particularly true for drivers who used the right lane mainly to allow passing of faster vehicles and not because they drove at very low speeds. The other major trend exhibited in this figure is the increase in the passing lane traffic split at lower traffic levels and vice versa, which is somewhat expected.

\section{Discussion in Regards to Driver Behavior}

In this section, the trends exhibited in the previous figures are analyzed in relation to drivers' lane selection (lane changing) behavior within the passing lane section. Those figures show that the percentage of short headways in the normal lane rose sharply in the first 0.25 mile. Half mile into the passing section, the percentage of short headways in the normal lane dropped to below the pre-passing lane value. Meanwhile, average travel speed of vehicles in this same lane continued to increase (until the 0.5-mile station). Those trends can be interpreted as follows: between the upstream and the 0.25-mile stations, aggressive drivers chose the left lane and begun speeding up. The moderate drivers (or majority of them) also chose the left lane in order to pass the slowest vehicles before they move to the passing lane. Between 0.25 mile and 0.5 mile, the moderate drivers shifted to the right lane upon passing the slower vehicles. This is supported by the dramatic drop in percent followers in the left lane. Further, Fig. 5 shows the highest percent of drivers were in the passing lane occurring at the 0.5 -mile station. By the time the traffic reached the 0.5-mile station, the aggressive drivers had been able to pass the moderate (and slowest vehicles) and the moderate drivers had been able to pass the slowest vehicles. Beyond that point, drivers began transitioning back gradually to the normal lane from the passing lane. This implies that, for this particular site, a passing lane length between 0.75 and 1.0 mile may prove to be adequate for all passing purposes.

\section{Discussion on Passing Lane Length}

In this research, passing maneuvers and lane changing were indirectly examined through the use of performance measures and volume lane split at successive locations within a passing lane section. Traffic volumes at the study site, though ranged between low and moderate traffic levels, are typical on two-lane highways in many rural states. For this case study site, it was evident that traffic performance became relatively stable beyond half a mile into the passing lane, which suggests that most passing maneuvers already took place before the 0.5 -mile station. Accordingly, it is logical to conclude that the actual length of the passing lane under study is well beyond the optimal length given traffic conditions prevalent at the study site.

\section{Concluding Remark}

This paper presents a case study investigation of traffic performance and lane utilization at a passing lane section on a rural two-lane highway in Montana. The analyses provided valuable insights which lead to better understand driver's lane selection and lane changing behavior within the passing lane section. While this case study involved a single passing lane site, the results presented in this 
paper are applicable to many similar passing lanes on rural two-lane highways.

\section{References}

Al-Kaisy, A.; Karjala, S. 2008. Indicators of Performance on Two-Lane Rural Highways: An Empirical Investigation, Transportation Research Record: Journal of the Transportation Research Board. DOI: http://dx.doi. org/10.3141/2071-11, 2071: 87-97.

American Association of State Highway and Transportation Officials (AASHTO). 2004. A Policy on Geometric Design of Highways and Streets, Washington, D.C.

Emoto, T.C.; May, A.D. 1988. Operational Evaluation of Passing Lanes in Level Terrain. FinalReport. Report UCBRR-88-13. Institute of Transportation Studies, University of California at Berkeley.

Gattis, J.; Bhave, R.; Duncan, L. 2006. Alternating Passing Lane Length, Transportation Research Record: Journal of the Transportation Research Board. DOI: http://dx.doi. org/10.3141/1961-03, 1961: 16-23.

Harwood, D.W.; Hoban, C.J. 1987. Low Cost Methods of Operational Improvements on Two-Lane Highways. Midwest Research Institute and Federal Highway Administration. Report No. FHWA/IP-87/2. Contract No. DTFH6182-C-00070.
Harwood, D.W.; St. John, A.D. 1984. Passing Lanes and Other Operational Improvements on Two-Lane Highways. Report No. FHWA-RD-85-028. Federal Highway Administration, U.S. Department of Transportation, Washington, D.C.

Transportation Research Board (TRB). 2000. Highway Capacity Manual. Fourth Edition. TRB, National Research Council, Washington, D.C.

Transportation Research Board (TRB). 2010. Highway Capacity Manual. Fifth Edition. TRB, National Research Council, Washington, D.C.

Woolridge, M.; Messer, C.; Heard, B. 2002. Design Criteria for Improved Two-Lane Section (Super 2). Texas Transportation Institute, Project 0-4064.

Van As, C. 2003. The Development of an Analysis Method for the Determination of Level of Service of Two-Lane Undivided Highways in South Africa. Project Summary, South African National Roads Agency, Limited. 